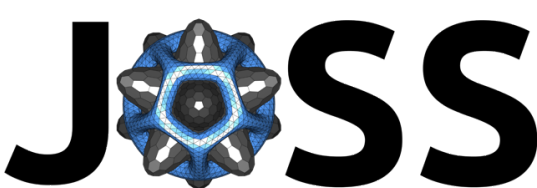

The Journal of Open Source Software

DOI: $10.21105 /$ joss. 01326

\title{
Phobos: A tool for creating complex robot models
}

\section{Kai von Szadkowski ${ }^{1}$ and Simon Reichel ${ }^{1}$}

1 German Research Center for Artificial Intelligence GmbH - Robotics Innovation Center, Bremen

\section{Software}

- Review ¿

- Repository ¿

- Archive [

Editor: George K. Thiruvathukal ¿

\section{Reviewers:}

- QCameronDevine

- @trallard

Submitted: 15 February 2019 Published: 07 January 2020

\section{License}

Authors of papers retain copyright and release the work under a Creative Commons Attribution 4.0 International License (CC-BY).

\section{Summary}

The rise of task specific requirements for robots leads to an increasing complexity of the affiliated system. To ensure a seamless, simulation based development of both hard- and software a virtual twin is necessary, which is able to reflect different aspects of the mechatronic system. Most description formats for these systems are based on XML (eXtensible Markup Language), which encodes the necessary data for simulation, e.g. mechanical structure, inertial data, visual and collision representations. However, the initial modeling or continuous refinement of the model based on design iterations, sensor and actor integration can be a time consuming, challenging task for the modeler. Also, tiny mistakes in the model description can lead to huge differences in the modeled system. With increasing model complexity the model files become even more difficult to debug.

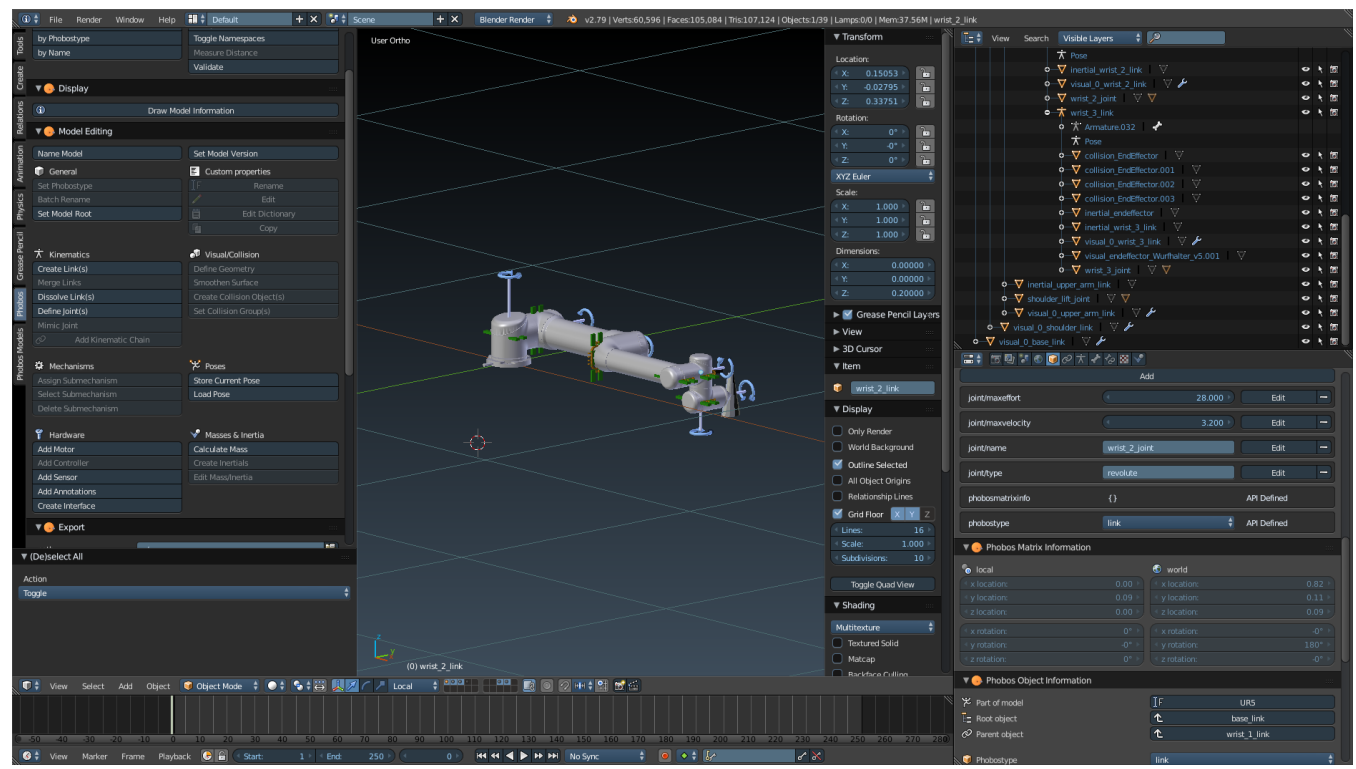

Figure 1: Universal Robots UR5 (https://www.universal-robots.com/products/ur5-robot/) within Phobos

Phobos is an add-on for the open-source 3D modeling software Blender that allows users to edit models of robots and their environments for robotics research and applications.

It integrates seamlessly in Blender's GUI and adds functionality for creating accurate representations of robotic systems with clearly defined kinematic chains, actuators and sensors in a WYSIWYG (What You See Is What You Get) environment.

To simplify common workflows further, all components of Phobos models in Blender can be annotated with custom data fields both individually or in groups. Such data can become 
part of exported model formats that support them (e.g. to define new types of sensors and other hardware devices) or kept for internal use, enabling the user to manipulate multiple components of a model using custom Python scripts. As Phobos is built on Blender's internal Python scripting API, it is easy to create scripts that work with both Blender- and Phobosspecific data.

Current features of Phobos include:

- support for a number of common description formats for robotic devices, such as:

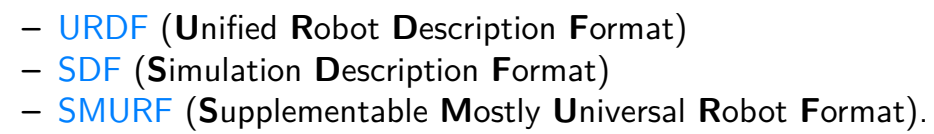

- visual representation of many different components, such as model links and joints, inertias, motors etc.

- hierarchical overview over the model

- useful batch tools, like renaming, reorientation of the model tree etc.

- automated mass and inertia calculation

- saving and loading of different model poses

- tools for maintaining your own model database

- creation of submodels which can be incorporated into other models

- definition of export configurations, where the high fidelity model is either used as a template or can be simplified

- internal checking of associated data for integrity

- an additional library containing Python examples for automatic model adaption

Furthermore, the user can tap into Blenders capabilities to visualize joint limits via the Pose Editing Mode, simplify associated visual and collision meshes or write Python scripts to modifiy the model objects. Since import and export are plugin-based, adding another format is as simple as adding a Python script to the respective subfolder. Phobos uses a Python dictionary in which all model information is stored for $\mathrm{I} / \mathrm{O}$ purposes, thus new developers do not have to program against Blender's representations of model elements.

Phobos was developed at the German Research Center for Artificial Intelligence's Robotics Innovation Center (DFKI-RIC in Bremen in collaboration with the University of Bremen's Robotics Group. The goal behind its development is to simplify the task of creating accurate descriptions of robotic systems in an intuitive and less error-prone way than editing them by hand in a text editor, and to provide an open-source alternative to exporting such models from proprietary CAD systems.

It has been used recently to provide the models for seriell-parallel hybrid robots modelling (Kumar, Szadkowski, Mueller, \& Kirchner, 2018).

Phobos can be found at Github ("Phobos GitHub Repository," 2019).

\section{Acknowledgements}

We acknowledge contributions from Stefan Rahms, Ole Schwiegert und Malte Langosz.

Phobos was carried out in the projects:

- LIMES, a collaboration between the DFKI Robotics Inno- vation Center and the University of Bremen, funded by the German Space Agency (DLR, Grant numbers: 50RA1218, 50RA1219) with federal funds of the Federal Ministry of Economics and Technology (BMWi) in accordance with the parliamentary resolution of the German Parliament. 
- D-RoCK, funded by the German Aerospace Center (DLR) with federal funds from the Federal Ministry of Education and Research (BMBF) (Grant Numbers: 01IW15001)

- TransFit, which is funded by the German Federal Ministry of Economics and Technology (BMWi) according to a resolution of the German Bundestag, grant no. 50RA1701, 50RA1702 and 50RA1703.

\section{References}

Kumar, S., Szadkowski, K. von, Mueller, A., \& Kirchner, F. (2018). HyRoDyn: A modular software framework for solving analytical kinematics and dynamics of series-parallel hybrid robots. In.

Phobos GitHub Repository. (2019). https://github.com/dfki-ric/phobos. 